\title{
RINTC PROJECT: INFLUENCE OF STRUCTURE-RELATED UNCERTAINTIES ON THE RISK OF COLLAPSE OF ITALIAN CODE- CONFORMING REINFORCED CONCRETE BUILDINGS
}

\author{
Paolo Franchin $^{1}$, Fabrizio Mollaioli ${ }^{1}$, Fabrizio Noto ${ }^{2}$ \\ ${ }^{1}$ Sapienza University of Rome \\ Dept. of Structures for Engineering and Architecture, via Gramsci 53, 00197 Rome, Italy \\ e-mail: paolo.franchin@uniroma1.it, fabrizio.mollaioli@uniroma1.it \\ ${ }^{2}$ METIS srl \\ via Emanuele Filiberto 207, 00185 Rome, Italy \\ e-mail: fabrizio.noto@gmail.com
}

Keywords: reinforced concrete, nonlinear static and dynamic analyses, lumped plasticity models, infill models, multi-stripe analyses, risk assessment.

\begin{abstract}
This paper reports on the results of an ongoing Research Project aimed at computing the risk of collapse in new buildings conforming to the Italian Seismic Design Code. Companion papers describe the overall Research Project, funded by the Italian Civil Protection Department (DPC), its different application areas (reinforced concrete, masonry, steel buildings, etc), the overall seismic risk calculation procedure and the ground motion selection process followed to identify the recorded ground motions used for the multi-stripe analyses for ten different ground motion intensities.
\end{abstract}

This papers describes the multivariate statistical model of the structure-related uncertainty developed with reference to reinforced concrete buildings, describing the variability of material properties as well as model error terms of the adopted response models for both RC members and masonry infills. The paper describes also the efficient sampling procedure adopted and discusses the results of the nonlinear analyses, both static and dynamic, carried out under different assumptions on the correlation structure for the selected reinforced concrete buildings (namely 6- and 9-storey moment resisting frame). 


\section{INTRODUCTION}

As described in the companion papers from the RINTC project, and in particular in [9][1], three sets of 3, 6 and 9-story moment-resisting RC frame buildings were designed to comply with the current Eurocode-aligned national structural design code and were subjected to seismic risk assessment via multiple-stripe analysis with conditional-spectrum selected motions. The buildings are all intended for residential use and are characterized by regularity in plan and elevation, with identical floor plans (floor beams and floor slabs), the only differences being in the column dimensions and reinforcement. Details about the geometry, loads, design process, inelastic modeling strategy and analyses results can be found in [1].

Analyses have been carried out for all cases accounting only for uncertainties on the action side, in terms of seismic hazard curve and ground-motion record-to-record variability. This paper describes the sub-set of analyses carried out by the authors to gauge the effect on the results of structure-related uncertainty, by which it is collectively indicated the variability of the structural materials as well as the epistemic uncertainty in the response models (model uncertainty). This exploration has been so far limited to the only site of Naples, out of the five considered in the project, soil category $\mathrm{C}$, and only for the 6- and 9-storey buildings. For length reasons, this paper presents the 6-story building configurations only.

\section{METHODOLOGY}

\subsection{Joint distribution model of the selected random vector}

As it regards model uncertainty, two models are used in the analysis of the selected RC buildings: the IMK response model, for the RC members, and an equivalent strut (concentric) model for the infills. The former, as anticipated, is characterized by a total of five parameters. Marginal predictive equations have been derived for these parameters by Haselton et al. The equations are based on the assumption that parameters have a lognormal distribution, and come in the form of an equation for the median, and a dispersion value. The model is marginal in the sense that no measure of statistical dependence among the parameters is provided. In the analyses reported in the previous sections, for all configurations, sites and soil conditions, median values have been used. In this paper, for the site of Naples and soil condition $\mathrm{C}$, the model parameters are modelled as lognormal variables based on Haselton et al equations, with the exception of the ultimate to yield moment ratio, that is taken deterministic equal to the median prediction. Two additional lognormal variables are used to describe material variability in RC members, and in particular concrete strength $f_{c}$ and steel yield stress $f_{y}$, thus, in sum, $4+2=6$ random variables are used for the response model of RC members (see Figure 1).

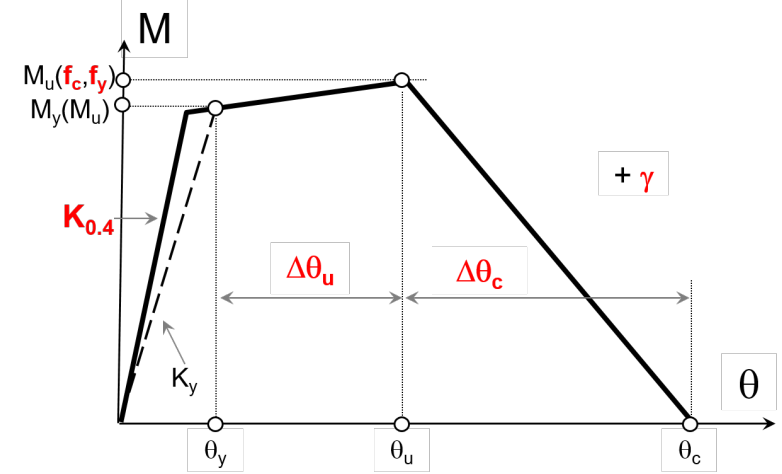

Figure 1: Trilinear IMK model used for hinges in RC member modeling: identification of random variables. 
The parameters of the infill model are five, peak strength, cracking, peak and residual strength displacements, and the strength reduction factor for openings. Thus, five random variables are needed for each equivalent strut.

In order to perform the analysis with account of the above uncertainties, two choices have been made. First, it has been chosen not to consider all possible combinations of ground motions and structure-related variables values, i.e. as in a so-called "extended IDA" framework. This has been judged computationally too onerous for the marginal increment in accuracy in the determination of the response parameters and final risk values. Instead, a one-to-one association of sets (realizations or samples) of structural parameters' values and ground motions has been done. This preserves the total number of inelastic response history analyses at 200 . The second choice regards the structure of the joint distribution of the random variables, which, in the absence of a joint predictive model, needs to be established based on expert judgement. It has been assumed what follows:

- Parameters within a beam or column member have a unique value, i.e. are perfectly correlated (joint lognormality is assumed based on marginal one, and as such, statistical dependence is completely described by a correlation model). This means that values of the IMK parameters and of $f_{c}$ and $f_{y}$ are the same in the two hinges of the same member;

- Similarly, for each infill panel, both equivalent struts are assigned a single set of parameters' values;

- For inter-member correlation, a simplified model has been adopted, whereby correlation is specified within each floor and amongst different floors, independently for each random variable, and independently of floor-to-floor distance (it is the same between roof and first floor, as it is, e.g., between second and third floor). This dependence structure is qualitatively justified based on the floor-wise erection of the buildings.

Under the above assumptions what remains to be established are the correlation values among different variables within each member, and the inter-member values, i.e. the intrafloor (superscript "sf" for "same floor") and inter-floor (superscript "df" for "different floor") values. While, as already said, Haselton et al [5] do not provide values for the intra-member correlations, and these are thus assumed based on judgement, at least for the displacement thresholds, these have been evaluated on the Sassun et al [11] experimental basis for the equivalent strut infill panel model. This is shown in Figure 2.

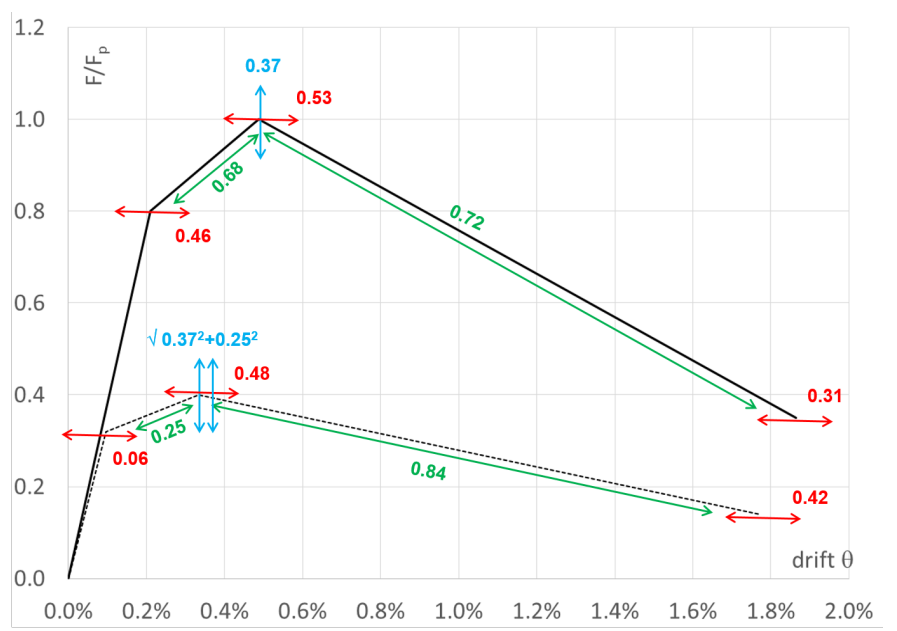

Figure 2: Diagonal strut model: dispersion (red for displacement, blue for forces) and correlation coefficients (green), assessed from the Sassun et al data base. Note that force for both full panels and panels with openings are normalized to full panel strength $\mathrm{F}_{\mathrm{p}}$. 
Table 1 and Table 2 summarize the logarithmic standard deviations (dispersions), intramember, intra-floor and inter-floor correlation coefficients, for the RC members and the infill panels (IP), respectively. Boldface values are based on experimental evidence, either provided by Haselton et al (Table 1) or assessed on purpose for this work (Table 2). The correlation and dispersion values for the IP equivalent strut model reported in the table are rounded with respect to those in Figure 2. The other assumed values only reflect the commonsense fact that, both in $\mathrm{RC}$ and masonry, increasing strength goes along with decreasing ductility.

\begin{tabular}{clcccccccc}
\hline Variable & $\sigma_{\text {In }}$ & $\rho_{x, f_{c}}$ & $\rho_{x, f_{y}}$ & $\rho_{x, \lambda}$ & $\rho_{x, k_{40}}$ & $\rho_{x, \Delta \theta_{u}}$ & $\rho_{x, \Delta \theta_{c}}$ & $\rho_{x, x}^{s f}$ & $\rho_{x, x}^{d f}$ \\
\hline$f_{c}$ & $\mathbf{0 . 2 0}$ & 1.0 & 0.0 & 0.0 & 0.0 & 0.0 & 0.0 & 0.6 & 0.0 \\
$f_{y}$ & $\mathbf{0 . 1 0}$ & 0.0 & 1.0 & 0.0 & 0.0 & 0.0 & 0.0 & 0.9 & 0.9 \\
$\lambda$ & $\mathbf{0 . 5 0}$ & 0.0 & 0.0 & 1.0 & 0.0 & 0.0 & 0.0 & 0.8 & 0.8 \\
$k_{40}$ & $\mathbf{0 . 3 8}$ & 0.0 & 0.0 & 0.0 & 1.0 & -0.5 & -0.5 & 0.8 & 0.8 \\
$\Delta \theta_{u}$ & $\mathbf{0 . 6 1}$ & 0.0 & 0.0 & 0.0 & -0.5 & 1.0 & 0.8 & 0.8 & 0.8 \\
$\Delta \theta_{c}$ & $\mathbf{0 . 7 2}$ & 0.0 & 0.0 & 0.0 & -0.5 & 0.8 & 1.0 & 0.8 & 0.8 \\
\hline
\end{tabular}

Table 1: Dispersion and intra-member correlation coefficients for the RC member model (values in bold typeface are based on data, the remaining ones are based on expert judgement).

\begin{tabular}{ccccccccc}
\hline Variable & $\sigma_{\text {In }}$ & $\rho_{x, f_{p}}$ & $\rho_{x, u_{c r}}$ & $\rho_{x, u_{p}}$ & $\rho_{x, u_{r}}$ & $\rho_{x, \rho_{o p}}$ & $\rho_{x, x}^{s f}$ & $\rho_{x, x}^{\text {df }}$ \\
\hline$f_{p}$ & $\mathbf{0 . 4 0}$ & 1.0 & -0.5 & -0.5 & -0.5 & 0.0 & 0.8 & 0.8 \\
$u_{c r}$ & $\mathbf{0 . 5 0}$ & -0.5 & 1.0 & $\mathbf{0 . 7}$ & $\mathbf{0 . 7}$ & 0.0 & 0.8 & 0.8 \\
$u_{p}$ & $\mathbf{0 . 5 0}$ & -0.5 & $\mathbf{0 . 7}$ & 1.0 & $\mathbf{0 . 7}$ & 0.0 & 0.8 & 0.8 \\
$u_{r}$ & $\mathbf{0 . 5 0}$ & -0.5 & $\mathbf{0 . 7}$ & $\mathbf{0 . 7}$ & 1.0 & 0.0 & 0.8 & 0.8 \\
$\rho_{\text {op }}$ & $\mathbf{0 . 2 5}$ & 0.0 & 0.0 & 0.0 & 0.0 & 1.0 & 0.8 & 0.8 \\
\hline
\end{tabular}

Table 2: Dispersion and intra-member correlation coefficients for the infill model (values in bold typeface are based on data, the remaining ones are based on expert judgement).

The final random vector, based on the previous assumptions, is joint lognormal, with median specified in [1] and covariance matrix:

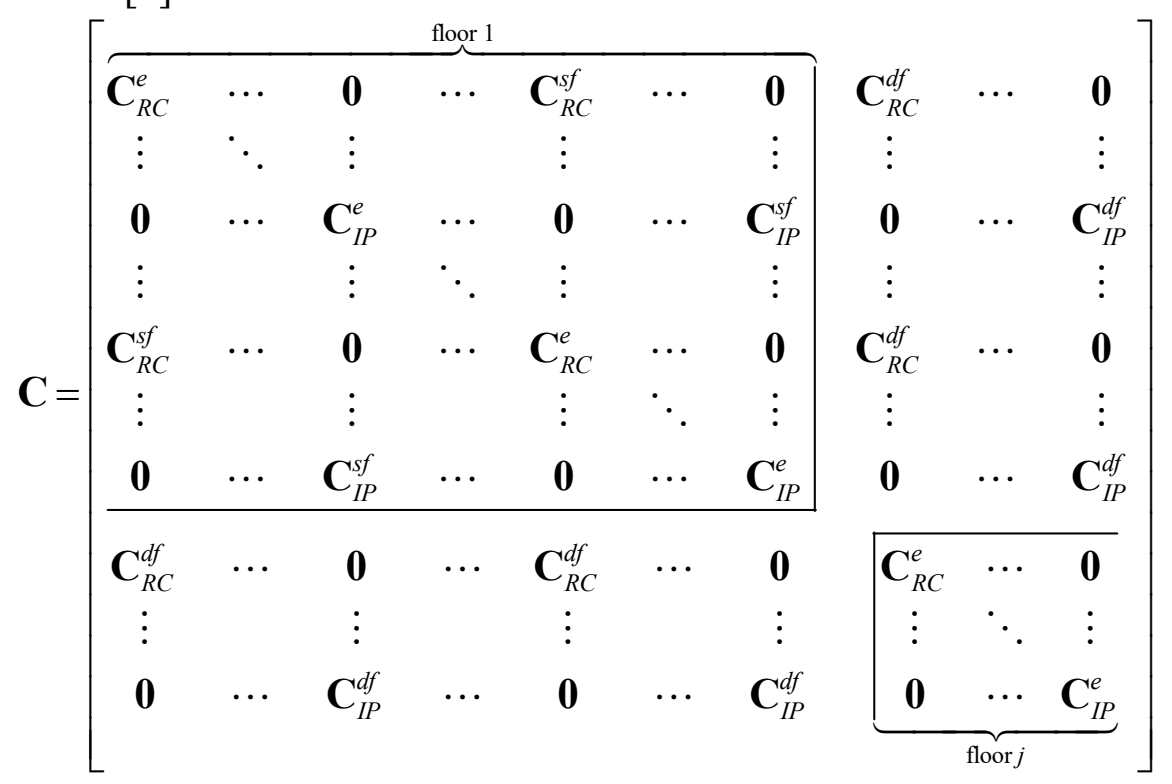


where:

$$
\begin{aligned}
\mathbf{C}_{R C}^{e} & =\left[c_{R C}^{e}\right]_{i_{1} i_{2}} \\
\mathbf{C}_{I P}^{e} & =\left[c_{I P}^{e}\right]_{i_{1} i_{2}}
\end{aligned}
$$

are the $6 \times 6$ and $5 \times 5$ intra-element covariance matrices of RC members and infill panels, respectively, given by the product $\mathbf{C}=\mathbf{D R D}$ of the respective correlation matrices $\mathbf{R}=[\rho]$ (columns 3 to 8 in Table 1 and 3 to 7 of Table 2, respectively) and the respective diagonal matrices $\mathbf{D}=\left[\sigma_{\mathrm{n}}\right]$ of logarithmic standard deviations (column 2 in both Table 1 and Table 2). The intra-floor (superscript "sf" for "same floor") and inter-floor (superscript "df" for "different floor") covariance matrices are given by:

$$
\begin{array}{r}
\mathbf{C}_{R C}^{s f}=\left[c_{R C}^{s f}\right]_{i_{1} i_{2}}=\left[c_{R C}^{e}\right]_{i_{1} i_{2}} \sqrt{\rho_{i_{1} i_{1}}^{s f} \rho_{i_{2} i_{2}}^{s f}} \\
\mathbf{C}_{R C}^{d f}=\left[c_{R C}^{d f}\right]_{i_{1} i_{2}}=\left[c_{R C}^{e}\right]_{i_{1} i_{2}} \sqrt{\rho_{i_{1} i_{1}}^{d f} \rho_{i_{2} i_{2}}^{d f}}
\end{array}
$$

and similarly for the infill panels.

\subsection{Efficient simulation of random samples}

The joint lognormal vector of random variables required to describe structure-related uncertainty according to the previous section:

$$
\mathbf{y}^{T}=\left\{\left(\mathbf{y}_{1,1}^{R C}\right)^{T} \cdots\left(\mathbf{y}_{1,1}^{I P}\right)^{T} \cdots\left(\mathbf{y}_{j, 1}^{R C}\right)^{T} \cdots\left(\mathbf{y}_{j, 1}^{I P}\right)^{T} \cdots\left(\mathbf{y}_{j, k}^{R C}\right)^{T} \cdots\left(\mathbf{y}_{j, k}^{I P}\right)^{T} \cdots\right\}
$$

has a size $\left(6 n_{R C}+5 n_{I P}\right) \times 1$ that easily reaches a few thousands of components (the indices $j$ and $k$ span the elements and floors, respectively). While direct sampling is still feasible according to the expression:

$$
\mathbf{y}=\exp \left[\mu_{\ln \mathbf{y}}(\mathbf{x})+\mathbf{D L u}\right]
$$

with $\mu_{\ln \mathbf{y}}(\mathbf{x})=\ln [\hat{\mathbf{y}}(\mathbf{x})], \mathbf{R}=\mathbf{L} \mathbf{L}^{\prime}$ and $\mathbf{u}$ is a vector of independent standard Gaussian variables, a more effective procedure has been devised, which uses the sums of independently generated short vectors (either $6 \times 1$ or $5 \times 1$, for RC members and infill panels, respectively) to reproduce the target covariance structure.

The $l$-th realization of the above vector is obtained as:

$$
\mathbf{y}_{j k l}=\hat{\mathbf{y}}_{j k l}(\mathbf{x}) \circ \exp \left(\boldsymbol{\varepsilon}_{j k l}\right)=\hat{\mathbf{y}}_{j k l}(\mathbf{x}) \circ \exp \left(\mathbf{c}_{x} \boldsymbol{\varepsilon}_{x_{j}}+\mathbf{c}_{y} \boldsymbol{\varepsilon}_{y_{k}}+\mathbf{c}_{z} \boldsymbol{\varepsilon}_{z_{l}}\right)
$$

where the symbol $\circ$ denotes the Hadamard or component-wise product of matrices, and $\boldsymbol{\varepsilon}_{j k l}, \boldsymbol{\varepsilon}_{x_{j}}, \boldsymbol{\varepsilon}_{y_{k}}, \boldsymbol{\varepsilon}_{z_{l}}$ are zero mean independent identically distributed Gaussian vectors of size $6 \times 1$ or $5 \times 1$, for RC members and infill panels, respectively, with covariance matrix either equal to $\mathbf{C}_{R C}^{e}$ or $\mathbf{C}_{I P}^{e}$. Not the same number of vectors is sampled for each type: for each simulation run (one value of index $l$ ), $n_{R C}+n_{I P}$ vectors $\boldsymbol{\varepsilon}_{x_{j}}, n_{\text {floors }}$ vectors $\boldsymbol{\varepsilon}_{y_{k}}$ and one vector $\boldsymbol{\varepsilon}_{z_{l}}$ are 
sampled. By calibrating the matrices $\mathbf{c}_{x}, \mathbf{c}_{y}, \mathbf{c}_{z}$ the correct overall covariance structure is obtained. In particular, setting the diagonal matrices $\mathbf{c}_{y}, \mathbf{c}_{z}$ to:

$$
\begin{aligned}
\mathbf{c}_{z} & =\left[\begin{array}{ccc}
\sqrt{\rho_{11}^{d f}} & \cdots & 0 \\
\vdots & \ddots & \vdots \\
0 & \cdots & \sqrt{\rho_{n n}^{d f}}
\end{array}\right] \\
\mathbf{c}_{y} & =\left[\begin{array}{ccc}
\sqrt{\rho_{11}^{s f}-\rho_{11}^{d f}} & \cdots & 0 \\
\vdots & \ddots & \vdots \\
0 & \cdots & \sqrt{\rho_{n n}^{s f}-\rho_{n n}^{d f}}
\end{array}\right]
\end{aligned}
$$

it can be easily shown that:

$$
\operatorname{cov}\left(\boldsymbol{\varepsilon}_{j_{1} k_{1} l}, \boldsymbol{\varepsilon}_{j_{2} k_{2} l}\right)=\mathbf{0}+\mathbf{0}+\mathbf{c}_{z}^{T} \mathbf{C}_{R C}^{e} \mathbf{c}_{z} \rightarrow \operatorname{cov}\left(\varepsilon_{i_{1} j_{1} k_{1} l}, \varepsilon_{i_{2} j_{2} k_{2} l}\right)=\left[c_{R C}^{e}\right]_{i_{1} i_{2}} \sqrt{\rho_{i_{1} i_{1}}^{d f} \rho_{i_{2} i_{2}}^{d f}}
$$

and:

$$
\operatorname{cov}\left(\boldsymbol{\varepsilon}_{j_{1} k l}, \boldsymbol{\varepsilon}_{j_{2} k l}\right)=\mathbf{0}+\mathbf{c}_{y}^{T} \mathbf{C}_{R C}^{e} \mathbf{c}_{y}+\mathbf{c}_{z}^{T} \mathbf{C}_{R C}^{e} \mathbf{c}_{z} \rightarrow \operatorname{cov}\left(\varepsilon_{i_{1} j_{1} k l}, \varepsilon_{i_{2} j_{2} k l}\right)=\left[c_{R C}^{e}\right]_{i_{i} i_{2}} \sqrt{\rho_{i_{1} i_{1}}^{s f} \rho_{i_{2} i_{2}}^{s f}}
$$

which coincide with equations $\mathrm{XX}$ and $\mathrm{YY}$ above. Finally, since the following condition must hold true:

$$
\operatorname{cov}\left(\boldsymbol{\varepsilon}_{j k l}, \boldsymbol{\varepsilon}_{j k l}\right)=\mathbf{c}_{x}^{T} \mathbf{C}_{R C}^{e} \mathbf{c}_{x}+\mathbf{c}_{y}^{T} \mathbf{C}_{R C}^{e} \mathbf{c}_{y}+\mathbf{c}_{z}^{T} \mathbf{C}_{R C}^{e} \mathbf{c}_{z}=\mathbf{C}_{R C}^{e}
$$

the last matrix is obtained as:

$$
\mathbf{c}_{x}=\mathbf{D} \sqrt{\mathbf{C W C}} \mathbf{D}^{T}
$$

where $\mathbf{W}=\mathbf{C}_{R C}^{e}-\left(\mathbf{c}_{y}^{T} \mathbf{C}_{R C}^{e} \mathbf{c}_{y}+\mathbf{c}_{z}^{T} \mathbf{C}_{R C}^{e} \mathbf{c}_{z}\right)$ must be definite positive for the covariance matric to be a valid one (a condition that is satisfied by using the inter-floor and intra-floor correlation values indicated in the previous tables).

\section{RESULTS}

\subsection{Nonlinear static analysis}

Nonlinear static analysis (NLSA) is employed to gain sensitivity on the model presented in the previous section. In particular, NLSAs have been performed in both X and Y directions, for 20 samples drawn from:

- A joint distribution with zero inter-member correlation

- A joint distribution with perfect inter-member correlation

- The joint distribution described in the previous section

Results are reported, with reference to the BF configuration only, for the sake of illustration, in the next Figure 3, where each row contains results for one of the three distribution models above, in the same top to bottom order, while the left and right column present the results for the $\mathrm{X}$ and $\mathrm{Y}$ direction, respectively. Similar results are obtained also for the IF and PF configurations. 

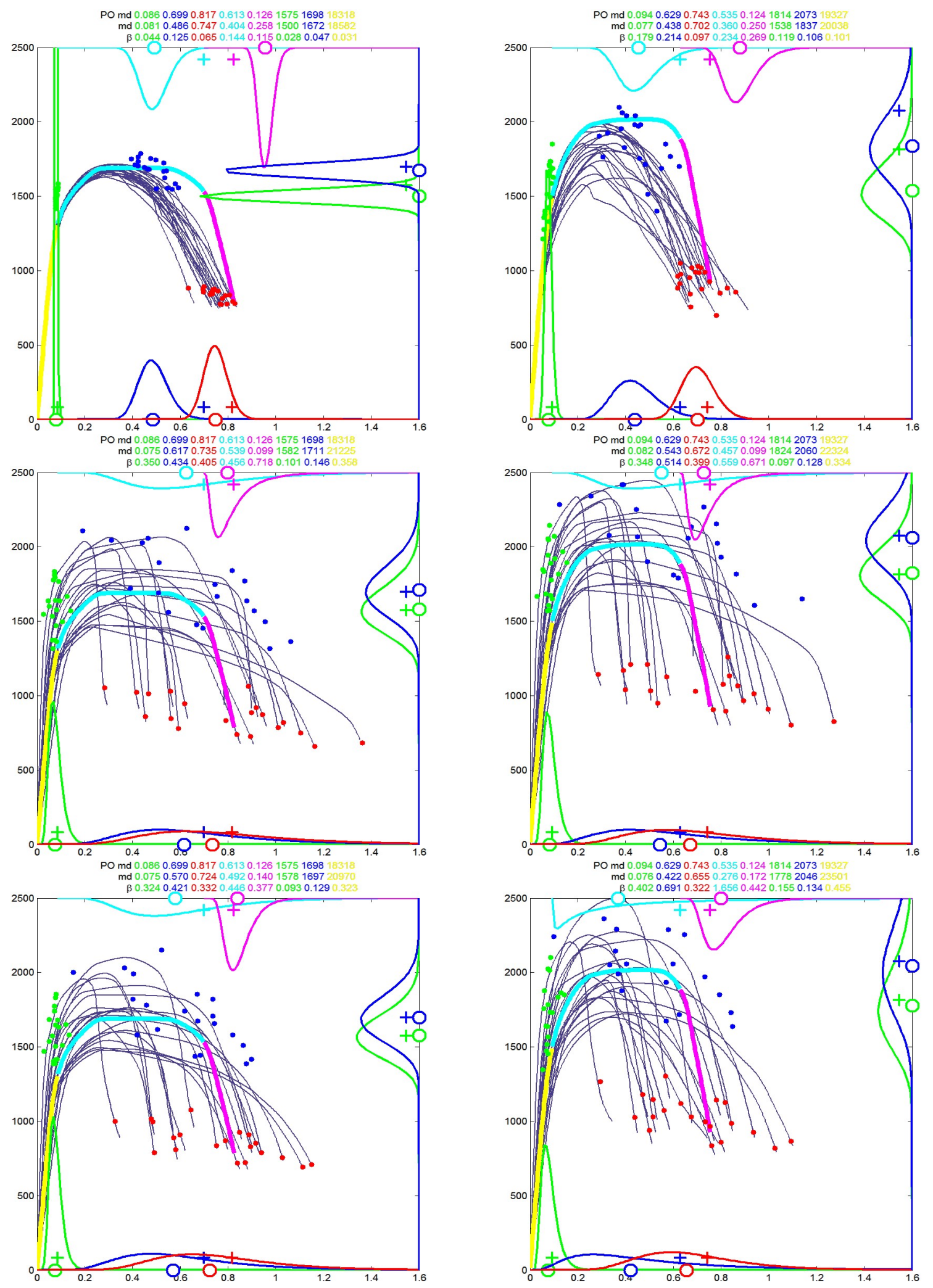

Figure 3: NLSA results (first mode distribution) in X (left) and Y (right) directions, BF configuration, under the three inter-member correlation assumptions ("zero", "perfect" and "assumed", from top to bottom). 
Without entering into the details, the thin lines represent individual NLSA runs, and green, blue and red dots mark the yield, peak and collapse (50\% force drop according to the adopted simplified global collapse criterion, see [1]) points on the (not shown, for clarity) trilinear fit to the curve. On the axes, lognormal PDFs fitted to both the displacement and force values obtained from these cloud of points are reported, and each plot is also completed by numerical values of median and dispersion with the same color code. A bold line with changing color denotes the capacity curve obtained with the median model.

The results allow to appreciate how the relatively high intra-floor and inter-floor correlation used leads to a model that is similar to a perfect correlation model. Also, the median model is providing in the last two cases an approximation of the median response of the analyses with consideration of structure-related uncertainties, while it is an upper bound for the "zero" inter-member correlation case. The latter is consistent and easily explained, because with high correlation, variations towards larger or smaller values (of strength, deformability, etc) happen to most members together, resulting in larger global variability reflected in the capacity curve. With independent members, it is highly likely instead that in each simulation one or more weak members are sampled that condition response causing a premature failure.
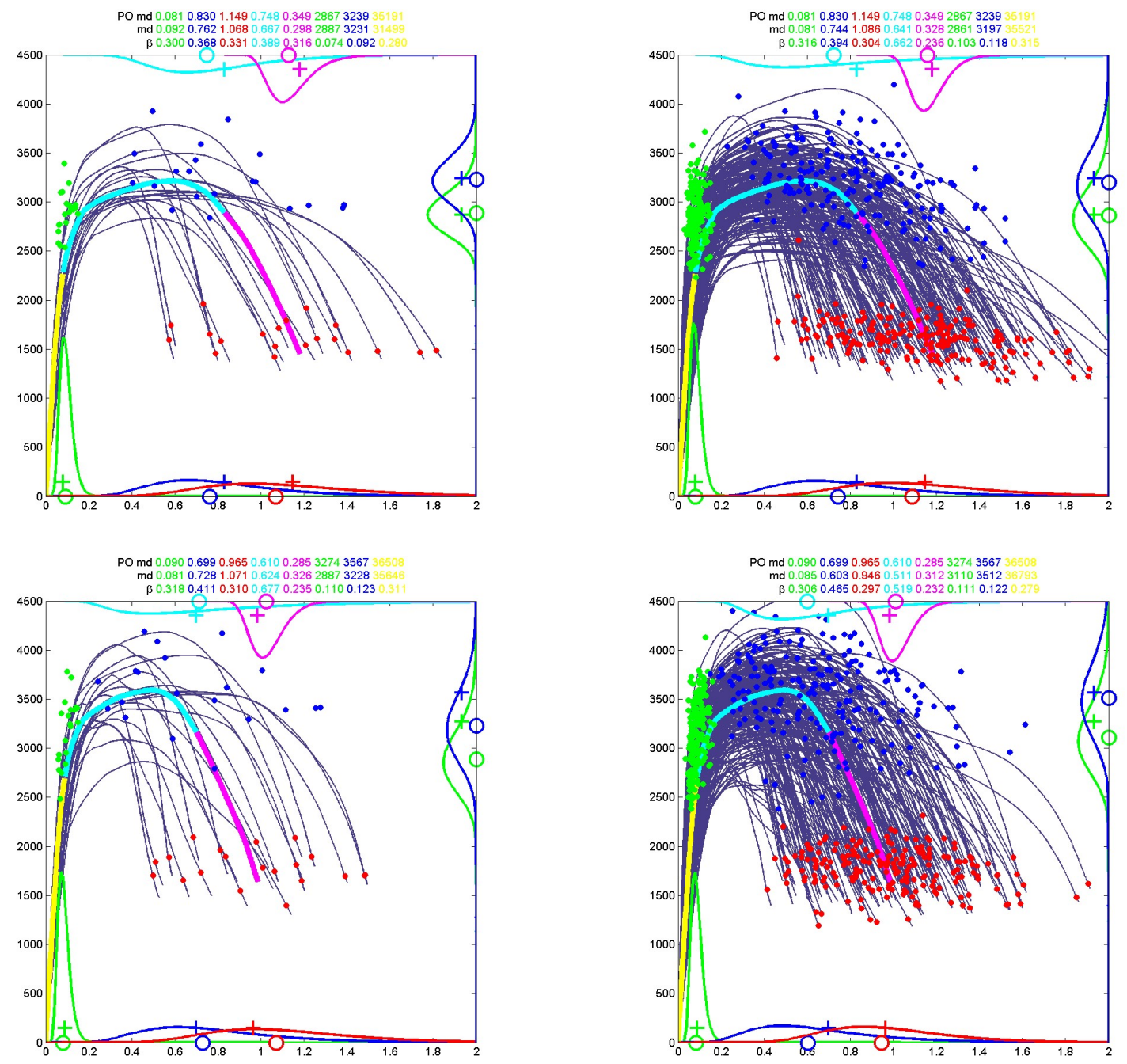

Figure 4: NLSA results (first mode distribution) in X (top) and Y (bottom) directions, BF configuration, under the assumed inter-member correlation assumption: effect of sample size on collapse capacity (20 vs 200 samples, left and right, respectively). 
Finally, Figure 4 shows a comparison of the results (fitted distribution and parameters) obtained with 20 or 200 samples, again only for the BF configuration for illustration's sake. The figure confirms that associating one-to-one a realization of the structure to each motion in a stripe, i.e. using 20 models, and using the same 20 random models for all the stripes (switching only the motion), provides a reasonable approximation.

\subsection{Nonlinear dynamic analysis}

Figure 5 reports the $\mathrm{D} / \mathrm{C}$ ratios (in terms of top floor displacement) for the $\mathrm{BF}$ configuration, for the X (left) and Y (right) directions, for both limit states. In particular, collapse is in the top row and damage is in the bottom one. The plots report both the individual run results, with markers aligned at each stripe intensity level, as well as the fitted conditional distributions. Blue markers and shaded PDFs are those for the median model analyses ([1]), shown for reference, while red denotes the results accounting for structure-related uncertainty. Figure 6 reports similar results for the IF configuration.
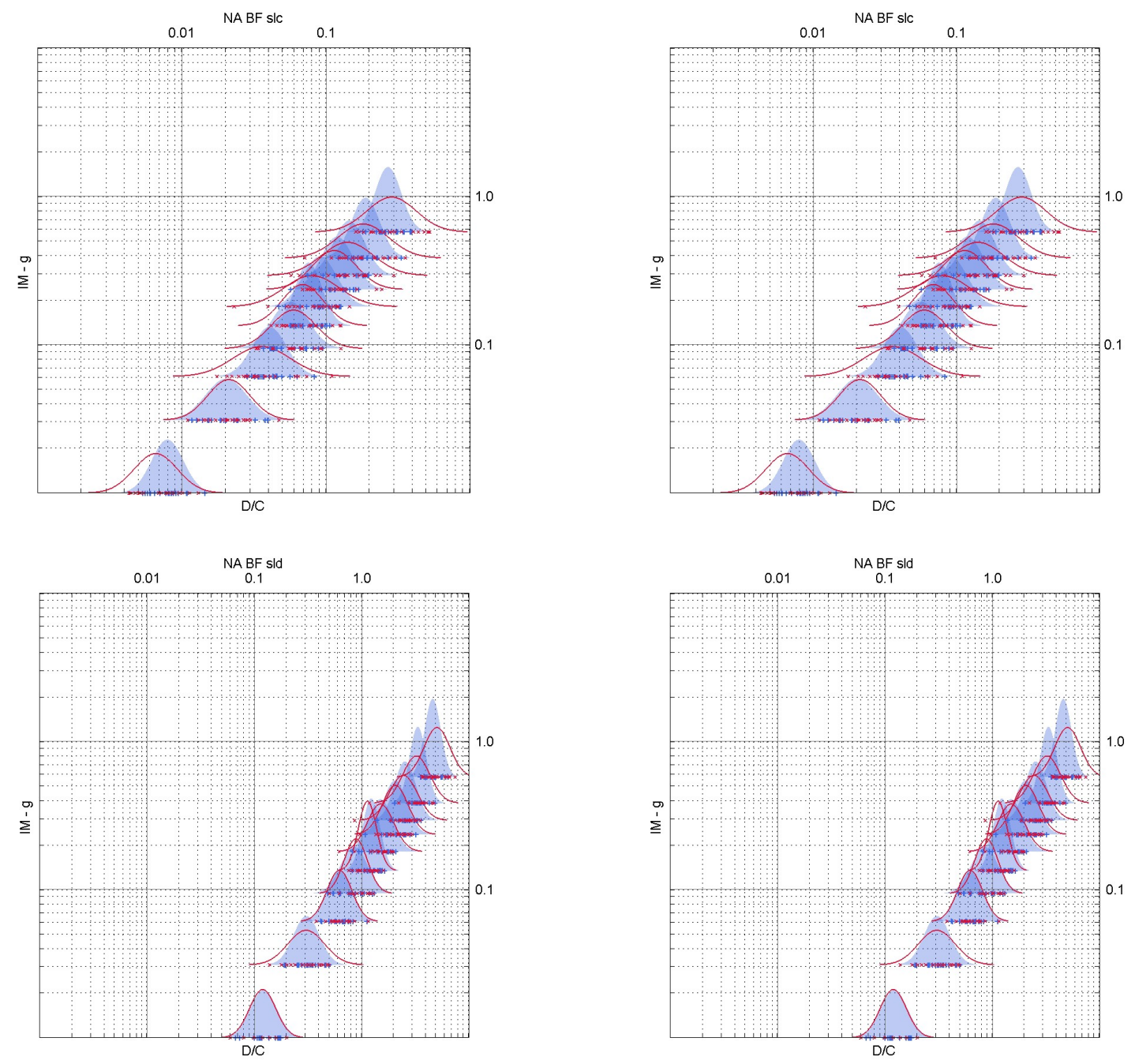

Figure 5: D/C ratios for the BF configuration (6-Story building in Naples, soil C): top row, collapse limit state, damage limit state in the bottom row, $\mathrm{X}$ direction in the left column, Y direction in the right column. Blue markers and light blue shade indicate individual run results and lognormal fit for the analyses with the median model, Red markers and line indicate corresponding results for the analysis with structure-related uncertainty. 

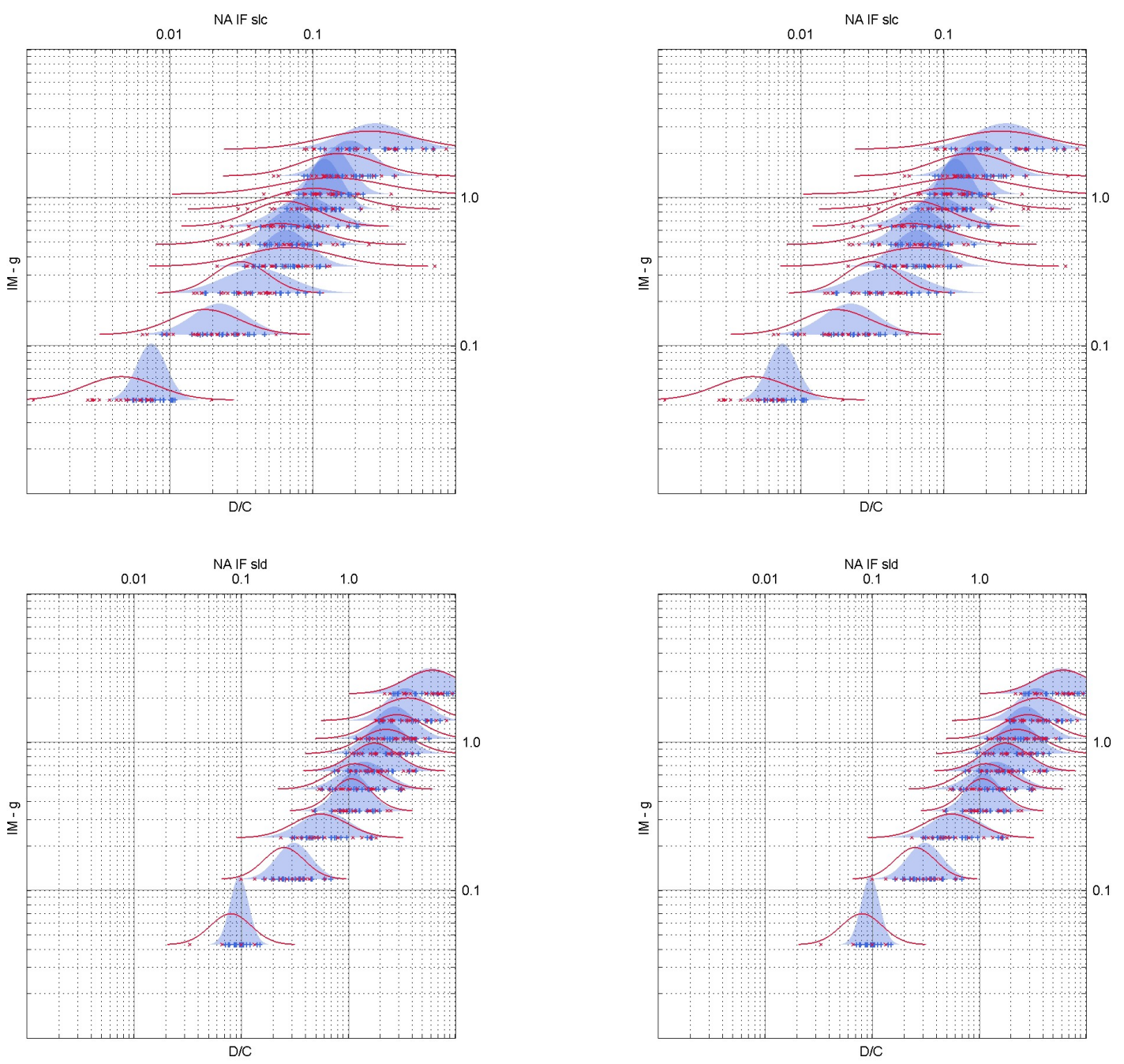

Figure 6: D/C ratios for the IF configuration (6-Story building in Naples, soil C): top row, collapse limit state, damage limit state in the bottom row, $\mathrm{X}$ direction in the left column, Y direction in the right column. Blue markers and light blue shade indicate individual run results and lognormal fit for the analyses with the median model,

Red markers and line indicate corresponding results for the analysis with structure-related uncertainty.

Results confirm that again the collapse $\mathrm{D} / \mathrm{C}$ ratios are well below 1.0 and those for the Damage Limit State are way above 1.0. It is reminded that the latter is defined as in NTC2008 by maximum InterStory Drift Ratios (IDRs) of 0.003 and 0.005 , if the model includes the infills, as in the IF and PF cases, or does not include them, as in the Bare Frame case, respectively. Even though the order of magnitude does not change, as expected, the dispersion of the $\mathrm{D} / \mathrm{C}$ ratios is quite heavily affected by the additional structure-related uncertainty, leading to a marked increase in the risk [9]. A better assessment of the induced change in terms of response can be obtained by inspecting the plots in Figure 7 (collapse limit state) and Figure 8 (damage limit state). The figures report, for all configurations (BF, IF and PF, from left to right), the cloud of $\mathrm{D} / \mathrm{C}$ points with abscissa equal to the $\mathrm{D} / \mathrm{C}$ ratio without structure-related uncertainty and the ordinate equal to the $\mathrm{D} / \mathrm{C}$ ratio with this uncertainty. In all cases the median is only slightly affected, with the possible exception of the IF configuration at collapse, and dispersion are quite large, ranging from 0.3 (BF) to 0.6 (IF and PF, where infill panels also play a role and total uncertainty increases) at collapse, and from 0.2 (BF) to 0.4 (IF) at the damage limit state. 

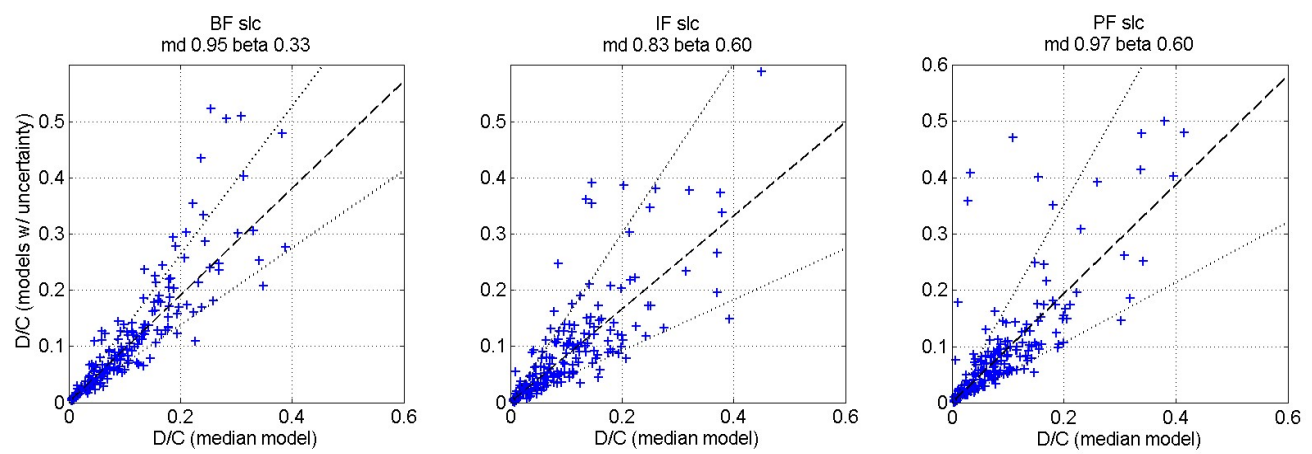

Figure 7: Collapse Limit State: D/C ratios of model with structure-related uncertainty versus model without it, for the same motion and for all configurations $(\mathrm{BF}, \mathrm{IF}$ and $\mathrm{PF})$.
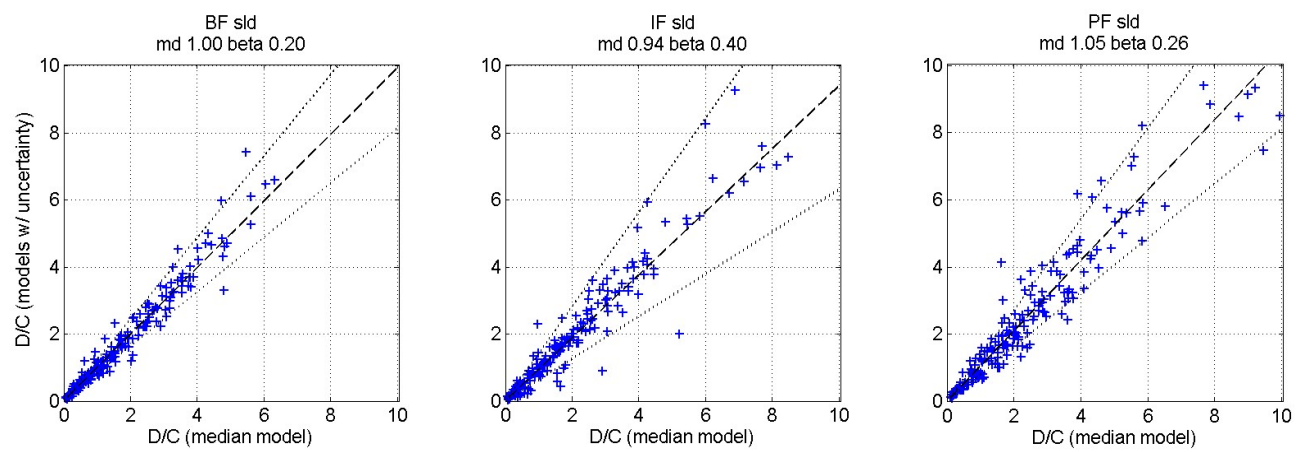

Figure 8: Damage Limit State: D/C ratios of model with structure-related uncertainty versus model without it, for the same motion and for all configurations (BF, IF and PF).

\section{CONCLUSIONS}

This paper is part of a set of papers presenting the results of an ongoing project on computing the implicit risk of seismic collapse (and damage) of buildings designed according to the current Italian design code NTC2008. This paper focuses on a set of 6-story RC buildings designed in one of the five sites of the project (Naples) and focuses on the assessment of the influence of structure-related uncertainty on the probabilistic seismic demand model established by nonlinear dynamic analysis. The joint distribution model adopted to describe this additional source of uncertainty (with respect to the action-related one, described through hazard curve and recorded ground motions) is presented and discussed. An efficient sampling procedure is also presented. The final results of the dynamic analyses confirm that this source of uncertainty is relevant and causes generally minor variations of the median response coupled with non-negligible increases of dispersion. The results is mostly due to the still relatively high model uncertainty associated with currently available response models in use.

\section{ACKNOWLEDGEMENTS}

The authors would like to acknowledge the financial support of the Italian Civil Protection Department, ReLUIS project 2014-2018 ( http://www.reluis.it/).

\section{REFERENCES}

[1] Camata, G., Celano, F., De Risi, M.T., Franchin, P., Magliulo, G., Manfredi, V., Masi, A., Mollaioli, F., Noto, F., Ricci, P., Spacone, E., Terrenzi, M., Verderame, G. RINTC project - Nonlinear dynamic analyses of Italian code-conforming reinforced concrete buildings for risk of collapse assessment. COMPDYN 2017 - 6th ECCOMAS Thematic 
Conference on Computational Methods in Structural Dynamics and Earthquake Engineering M. Papadrakakis, M. Fragiadakis (eds.) Rhodes Island, Greece, 15-17 June 2017.

[2] Decanini L.D., Fantin G.E., Modelos simplificados de la mamposteria incluida en porticos. Caracteristicas de rigidez y resistencia lateral en estado limite, Jornadas Argentinas de Ingenieria Estructural, Buenos Aires, Argentina, 1986, Vol.2, pp.817-836 (in Spanish), 1986.

[3] Decanini, L., Mollaioli, F., Mura, A., Saragoni R., Seismic performance of masonry infilled R/C frames, Proc.13th WCEE, Paper 165, Vancouver, B.C., Canada, August 1-6, 2004.

[4] Decanini L., Liberatore L., Mollaioli F. , Strength and stiffness reduction factors for infilled frames with openings, Earthquake Engineering and Engineering Vibration, 13(3): 437-454, September, 2014.

[5] Haselton, C.B., Liel, A.B., Taylor Lange, S. \& Deierlein, G.G., Beam-Column Element Model Calibrated for Predicting Flexural Response Leading to Global Collapse of RC Frame Buildings, PEER report 2007/03, 2008.

[6] Haselton, C. B., Liel, A. B., \& Deierlein, G. G., Simulating structural collapse due to earthquakes: model idealization, model calibration, and numerical solution algorithms. Computational Methods in Structural Dynamics and Earthquake Engineering (COMPDYN),2009.

[7] Ibarra, L. F., \& Krawinkler, H., Global collapse of frame structures under seismic excitations. Rep. No. TB 152, The John A. Blume Earthquake Engineering Center, 2005.

[8] Ibarra, L. F., Medina, R. A., \& Krawinkler, H., Hysteretic models that incorporate strength and stiffness deterioration. Earthquake engineering \& structural dynamics, 34(12), 1489-1511,2005.

[9] Iervolino, I., A. Spillatura, P. Bazzurro, RINTC Project - Assessing the (implicit) seismic risk of code-conforming structures in Italy. COMPDYN 2017 - 6th ECCOMAS Thematic Conference on Computational Methods in Structural Dynamics and Earthquake Engineering M. Papadrakakis, M. Fragiadakis (eds.) Rhodes Island, Greece, 1517 June 2017.

[10] McKenna, F. (2011) “OpenSees: a framework for earthquake engineering simulation", Computing in Science \& Engineering $13.4 \quad$ (2011): $58-66$ (http://opensees.berkeley.edu).

[11] Sassun, K., Sullivan, T. J., Morandi, P., Cardone, D., Characterising the in-plane seismic performance of infill masonry. Bulletin of the New Zealand Society for Earthquake Engineering, Vol. 49, No. 1,100-117, 2016. 